\title{
Linear and non-linear analysis of composite plates using guided acoustic waves
}

H. Alnuaimi, U. Amjad, P. Russo, V. Lopresto, T. Kundu

H. Alnuaimi, U. Amjad, P. Russo, V. Lopresto, T. Kundu, "Linear and non-linear analysis of composite plates using guided acoustic waves," Proc. SPIE 10972, Health Monitoring of Structural and Biological Systems XIII, 109720X (1 April 2019); doi: 10.1117/12.2513783

Event: SPIE Smart Structures + Nondestructive Evaluation, 2019, Denver, Colorado, United States 


\title{
Linear and non-linear analysis of composite plates using guided acoustic waves
}

\author{
H. Alnuaimi ${ }^{\mathrm{a}}$, U. Amjad ${ }^{\mathrm{a}}$, P. Russo ${ }^{\mathrm{b}}$, V. Lopresto ${ }^{\mathrm{c}}$ and T. Kundu ${ }^{\mathrm{a}, \mathrm{d}}$
}

${ }^{a}$ Department of Civil and Architectural Engineering and Mechanics, University of Arizona, Tucson, Arizona 85721, USA

${ }^{\mathrm{b}}$ Institute for Polymers, Composites and Biomaterials, National Research Council , Via Campi

Flegrei 34, Pozzuoli, 80078 Naples

${ }^{\mathrm{c}}$ Department of Chemical, Materials and Production, University of Naples Federico II, P.le Tecchio 80, 80125 Naples

d Aerospace and Mechanical Engineering Department, University of Arizona, Tucson, Arizona 85721, USA

\begin{abstract}
Guided acoustic wave techniques have been found to be very effective for damage detection. In this investigation Lead Zirconate Titanate (PZT) transducers are used to generate guided acoustic waves for structural health monitoring of a variety of composite specimens. Multiple sets of composite plate specimens are inspected for impact induced damage detection using PZT transducers. Composite samples are divided into two groups for comparative studies i.e. glass fiber composites and basalt fiber composites. They are damaged by impactors having different levels of impact energy. A chirp signal is excited and propagated through the specimens in a single sided excitation/detection setup to investigate the damages induced by impacts of varying intensity. Signal processing of the recorded signals for damage analysis involved both linear and nonlinear analyses. Linear ultrasonic analysis such as change in the time-of-flight of the propagating waves, Fast Fourier Transform and S-Transform of the recorded signals were tried out while the nonlinear ultrasonic analysis involved the Sideband Peak Count or the SPC technique.
\end{abstract}

Keywords: Guided ultrasonic waves, S-Transform (ST), Damage detection, Fast Fourier Transform (FFT), Side band peak count (SPC), Lead Zirconate Titanate (PZT), Non-Destructive Testing (NDT), Time of flight (TOF)

\section{Introduction}

In recent years the use of guided acoustic waves for non-destructive evaluation (NDE) has become increasingly popular for analyzing composite plates. It is often difficult to extract features for monitoring impact damages in composite plates using only traditional linear techniques therefore, this study will present both linear ${ }^{[1]}$ and non-linear methods ${ }^{[2]}$ of analysis. Guided acoustic waves are widely used for NDE to extract features for detecting and monitoring damages ${ }^{[1-10]}$. Multiple sets of composite plates are fabricated and examined by generating guided ultrasonic waves using Lead Zirconate Titanate (PZT) transducers. The composite plates are fabricated using thermoplastic resins due to their growing popularity in many fields, such as aerospace structures and marine structures. 


\section{Experimental Procedure}

\subsection{Materials}

The thermoplastic composite plates examined in this study are comprised of two types of reinforcing fibers glass and basalt fibers. Each plate has 18 plies of reinforcing material which make up 50\% of the samples by weight. A total of 6 sets of samples were analyzed. Each set has 3 samples giving a total of 18 samples. The 3 samples in a set are impacted with varying degrees of impact energy. 3 sets are reinforced with glass fibers and 3 sets are reinforced with basalt fibers; however, none of the sets have identical properties. The composite plates have a length of $20 \mathrm{~cm}$ a width of $10 \mathrm{~cm}$ and a thickness of $3 \mathrm{~mm}$. the densities of the samples and impact energies are summarized in Table.1 (Glass Fiber Samples) and Table. 2 (Basalt Fiber Samples).

Table 1: Summary of Glass Fiber Samples

\begin{tabular}{cccc}
\hline Glass Set & Number of Samples & Density g/cc & Impact Energy \\
\hline A & 3 & 1.72 & 0J (no impact), 20J, 30J \\
B & 3 & 1.72 & $10 \mathrm{~J}, 20 \mathrm{~J}, 30 \mathrm{~J}$ \\
C & 3 & 1.84 & $10 \mathrm{~J}, 20 \mathrm{~J}, 30 \mathrm{~J}$
\end{tabular}

Table 2: Summary of Basalt Fiber Samples

\begin{tabular}{cccc}
\hline Basalt Set & Number of Samples & Density g/cc & Impact Energy \\
\hline $\mathbf{X}$ & 3 & 1.94 & $10 \mathrm{~J}, 20 \mathrm{~J}, 30 \mathrm{~J}$ \\
$\mathbf{Y}$ & 3 & 1.82 & $10 \mathrm{~J}, 20 \mathrm{~J}, 30 \mathrm{~J}$ \\
& 3 & 1.82 & $10 \mathrm{~J}, 20 \mathrm{~J}, 30 \mathrm{~J}$ \\
\hline
\end{tabular}

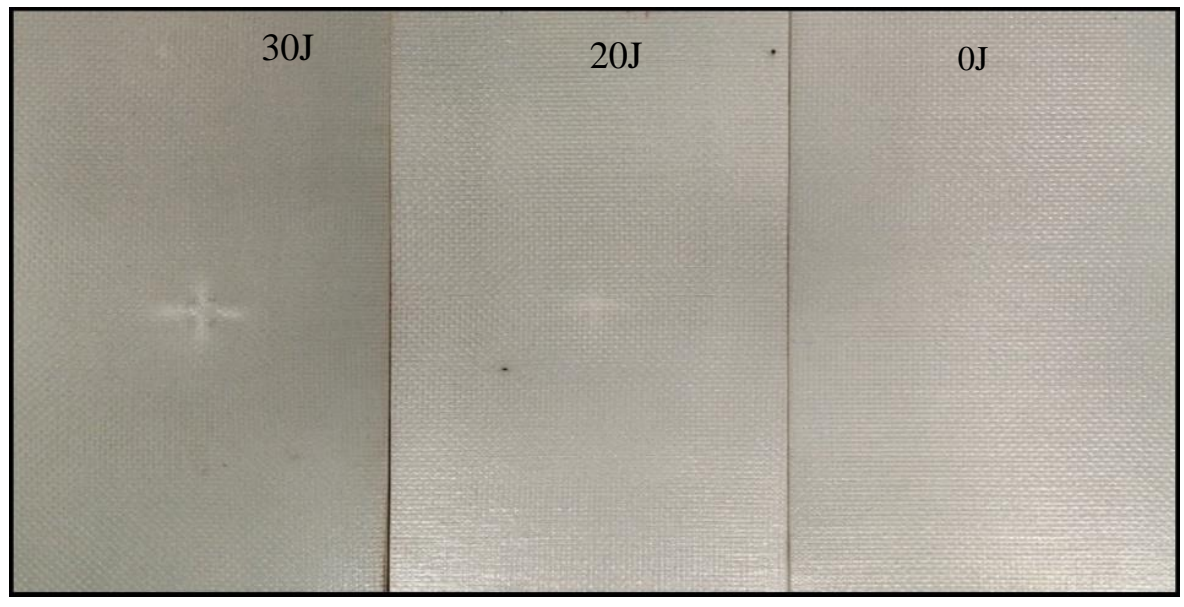

Figure 1: Photo of Glass Fiber Samples with Varying Impacts

All sets of composite plates are impacted at the center of the samples with varying degrees of impact energy. A single set of glass fiber samples are shown in Figure.1. It can be seen in Figure. 1 that the impact energy increases from 
right to left. Additionally, a set of basalt fiber samples are shown in Figure. 2. Comparing these two sets of samples one can conclude that different failure mechanisms cause these damages in the two sets of samples although they are impacted in the same manner.

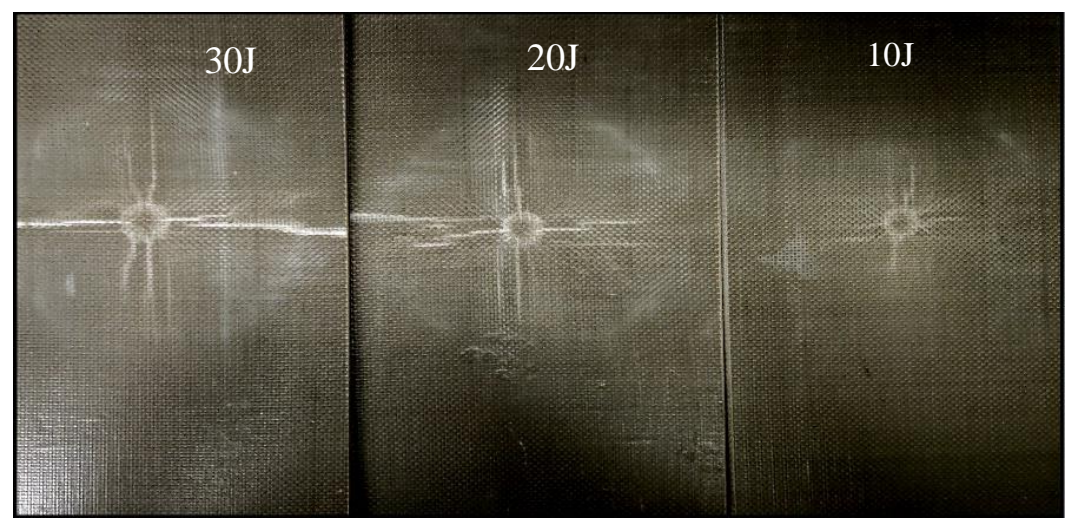

Figure 2: Photo of Basalt Fiber Samples with Varying Impacts

\subsection{Instrumentation and Methods}

The instrumentation and methods used in this study is summarized in Figure. 3. A computer controlled arbitrary function generator produces an electric pulse that is converted to an ultrasonic pulse by the transducer (excitation). The ultrasonic pulse then propagates through the composite plate specimen. At the receiving end, the ultrasonic pulse is detected and then converted back to an electric signal by a second transducer (detection). The PZT transducers that were used for this experiment ${ }^{[5-6]}$ have a diameter of $3.50 \mathrm{~cm}$ and a thickness of $0.02 \mathrm{~cm}$. The transducers are attached in direct contact with the composite samples when exciting and receiving the signal. For data acquisition, longitudinal guided wave modes are generated and propagated through the samples. TiePie engineering handy scope (HS3) is used as the arbitrary function (wave form) generator and transient recorder. The transient recorder has two channels that can record signals with a sampling frequency of $100 \mathrm{MHz}, 8$ bit resolution, and a memory of 131060 samples per channel. The arbitrary function generator is connected to a standard notebook PC that is used to log the data. The interfacing between the arbitrary function generator and synchronized transient recorder is programmed using LabVIEW@.

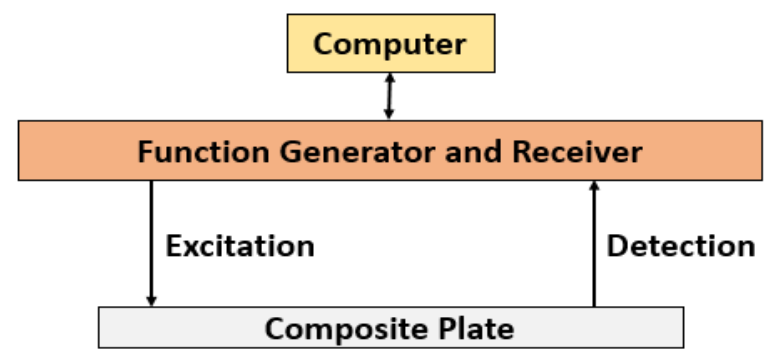

Figure 3: Schematic Diagram of the Experimental Setup

\subsection{Methods}

As explained earlier, data acquisition is done in transmission mode. A chirp signal is excited in a range from $50 \mathrm{kHz}$ to $250 \mathrm{kHz}$. In Figure. 4 a transient response is show for the 0J impact energy sample of glass set A (see Table 1). Similarly, responses for all samples are recorded and processed. Transient signals are normalized to 1 for better comparative studies. 


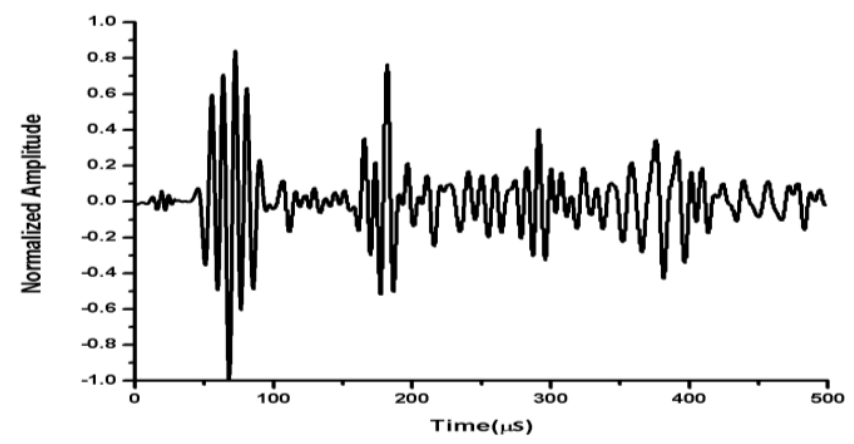

Figure 4: Transient Response of Glass Fiber Set A with O J Impact Energy

\section{Results and Discussion}

\subsection{Linear Analysis}

\subsubsection{Fast Fourier Transform}

A fast Fourier transform (FFT) is an algorithm that samples a signal over a period and divides it into its frequency components. In Figures 5 and 6, FFT's of three transient responses for sets A, B and C of glass fiber samples and for sets $\mathrm{X}, \mathrm{Y}$ and $\mathrm{Z}$ of basalt fiber samples are presented, respectively. Intensity changes are compared by normalizing all data to that corresponding to the sample impacted by the lowest impact energy.
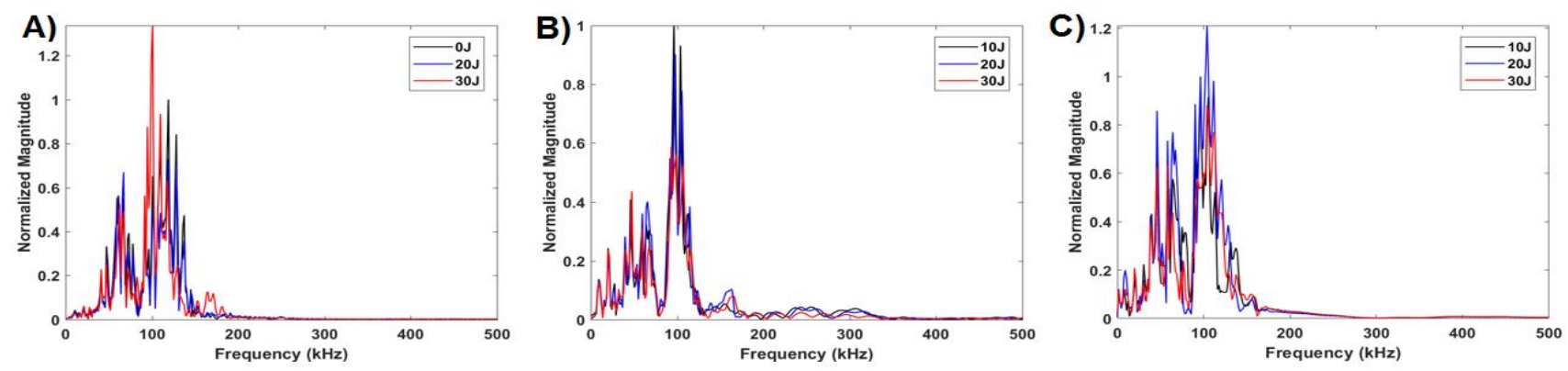

Figure 5: FFTs of All Glass Fiber Samples
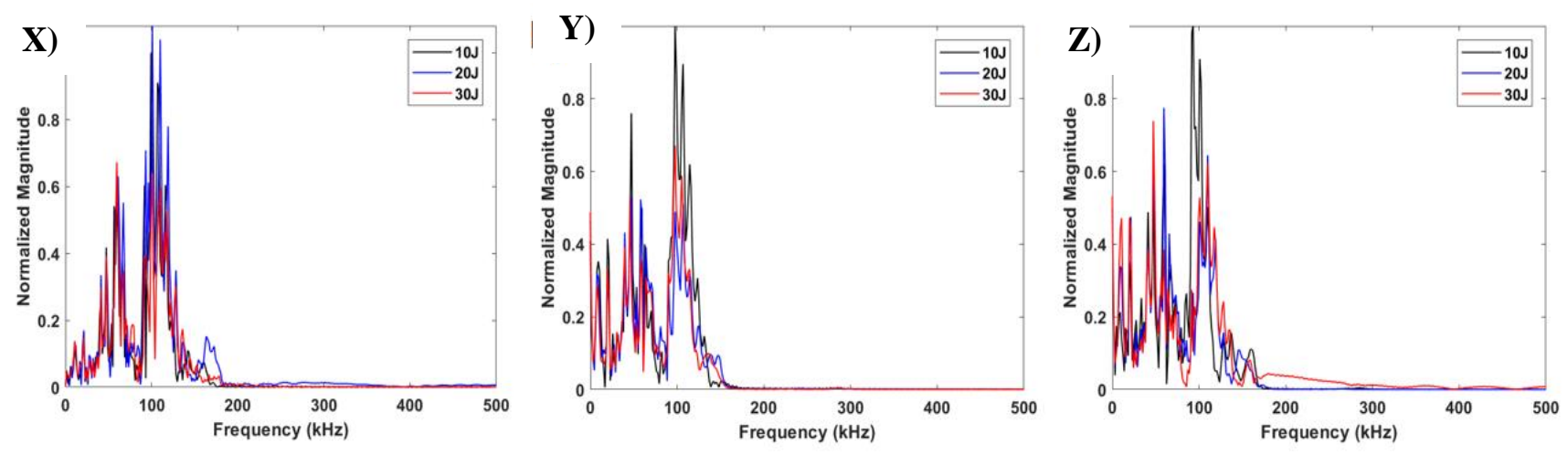

Figure 6: FFTs of All Basalt Fiber Samples 
Clearly, the magnitudes of the peaks and frequency response change with varying impact energy. The FFTs, used in this study to perform the nonlinear ultrasonic analysis based on the Sideband Peak Count (SPC) that will be discussed later, are also useful in determining how sensitive the samples are to specific frequencies.

\subsubsection{S-Transform}

The S-Transform is a combination of both Short Time Fourier Transform and Continuous Wavelet Transform. The S-Transform of a signal can be seen as a modified Short Time Fourier Transform with a Gaussian window of varying width and height as a function of frequency. It can also be interpreted as a modified wavelet transform (WT) with the phase correction in the mother wavelet. This modified wavelet ignores the wavelet's admissibility criterion of having the zero mean and, hence, cannot be considered as a Continuous Wavelet Transform.
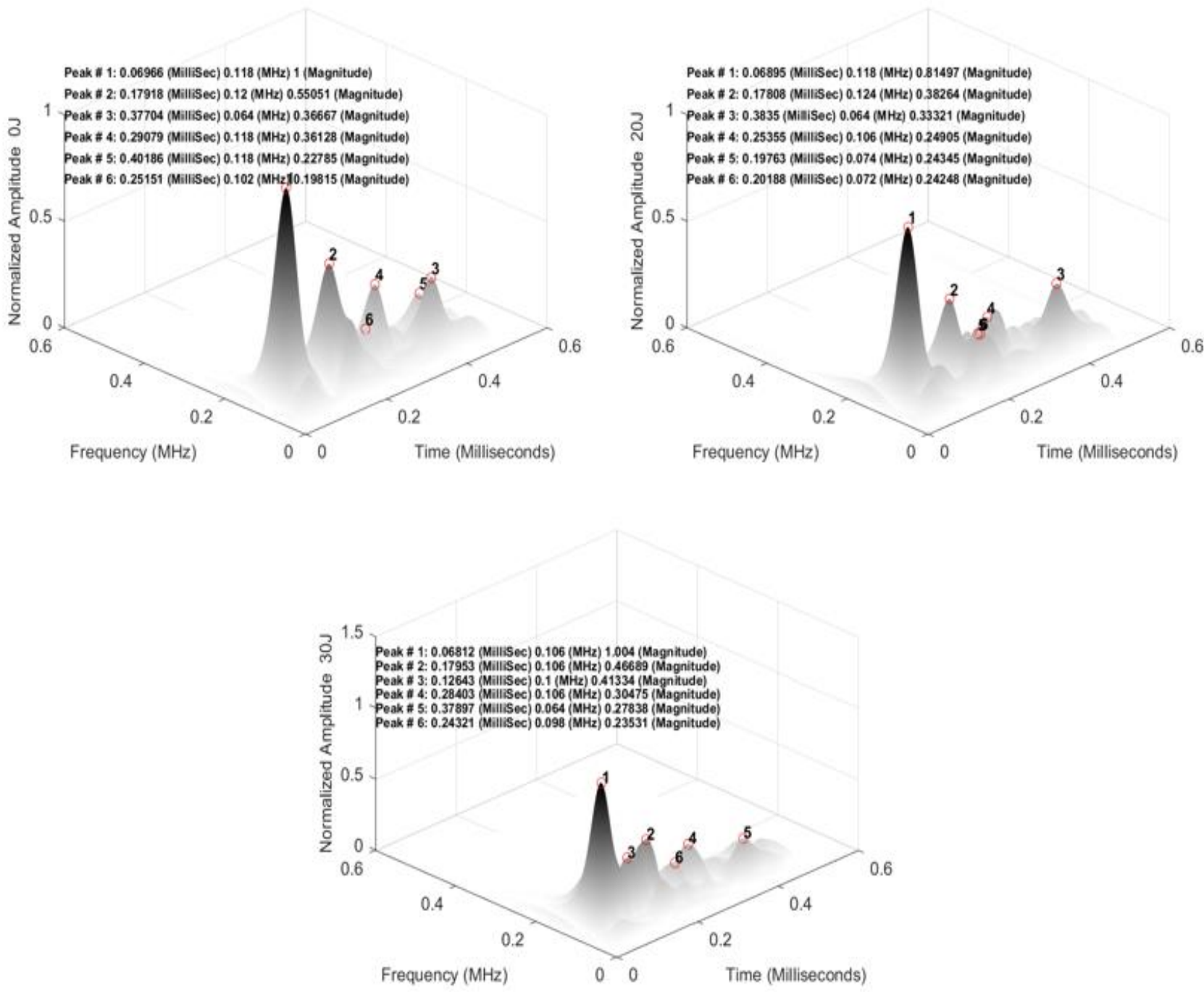

Figure 7: S-Transforms of Glass Fibers Set A

The Different peaks of the S-Transform plots shown in Figure. 7 represent the wave packets that are present in the transient signal. In details, the top left plot of Figure. 7 is obtained from the transient signal that can be seen in Figure. 4. These different peaks in the S-Transform plots are monitored and changes in TOF, frequency, and magnitude of the peaks are summarized and shown in Table. 3 . All plot magnitudes are normalized with respect to the values obtained from the sample impacted by the lowest impact energy. 
Table 3: Highest Peak values from S-Transform plots for Glass Fibers Set A

\begin{tabular}{cccc}
\hline Sample & Frequency (MHz) & Time (Millisecond) & Magnitude \\
\hline 0J & 0.118 & 0.06966 & 1 \\
20J & 0.118 & 0.06895 & 0.81497 \\
30J & 0.106 & 0.06812 & 1.004 \\
\hline
\end{tabular}

Data of S-Transform plots for sets B and C, not shown here, are summarized in Tables 4 and 5, respectively. The tables show information obtained from the most dominant peak for each sample that has approximately the same frequency. By examining the dominant peaks at a frequency, any shift in time of that peak can be attributed to the effect of the varying impact energy.

Table 4: Highest Peak Data from S-Transforms of Glass Fibers Set B

\begin{tabular}{cccc}
\hline Sample & Frequency (MHz) & Time (Millisecond) & Magnitude \\
\hline 10J & 0.1 & 0.0751 & 1 \\
20J & 0.102 & 0.07281 & 0.96313 \\
30J & 0.102 & 0.0717 & 0.84099 \\
\hline
\end{tabular}

Table 5: Highest Peak Data from S-Transforms of Glass Fibers Set C

\begin{tabular}{cccc}
\hline Sample & Frequency (MHz) & Time (Millisecond) & Magnitude \\
\hline 10J & 0.102 & 0.06194 & 1 \\
20J & 0.108 & 0.07317 & 1.4826 \\
30J & 0.108 & 0.06868 & 1.1836 \\
\hline
\end{tabular}



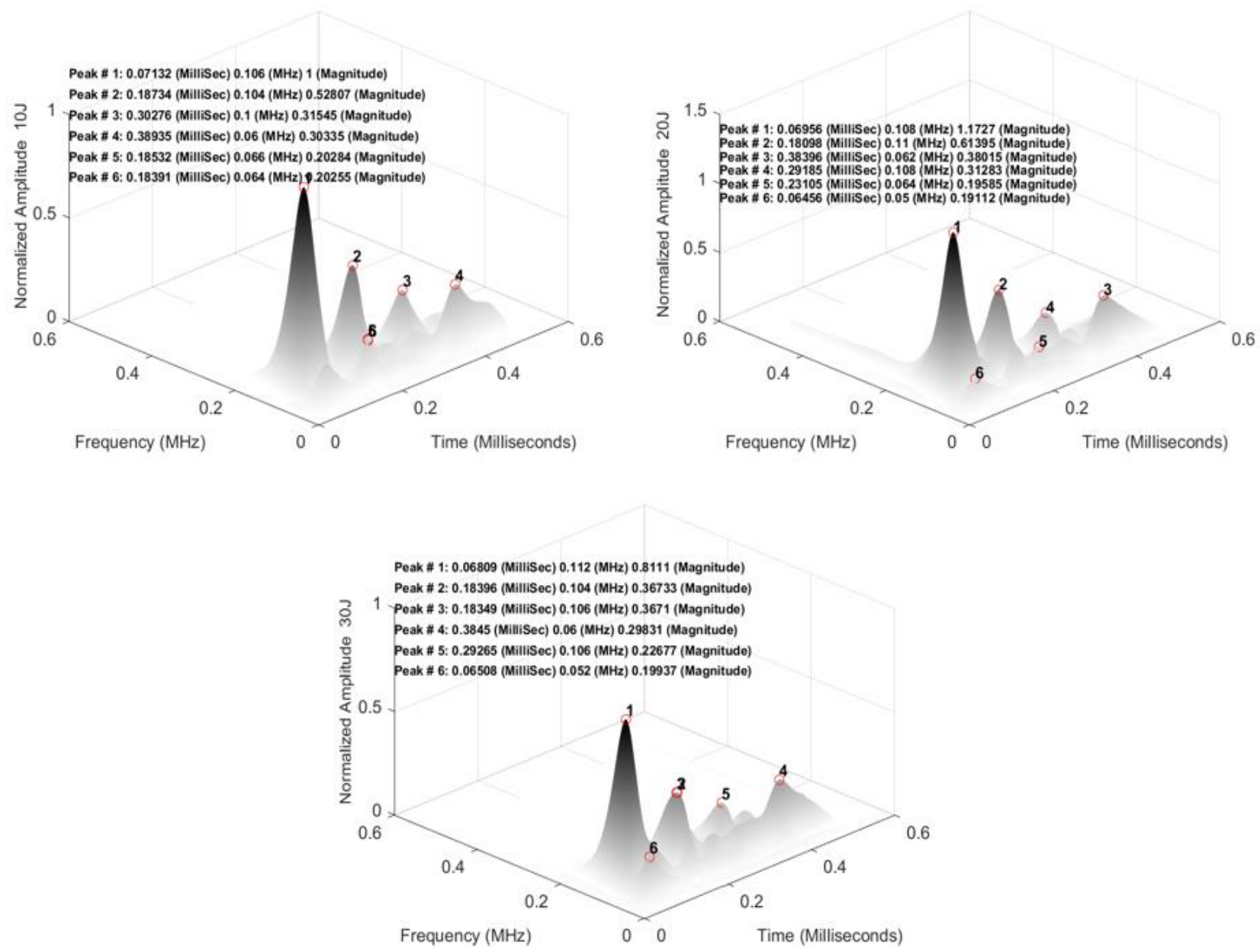

Figure 8: S-Transforms of Basalt Fibers Set X

The S-Transform plots of the basalt fiber (set X) samples are shown in Figure. 8. As before, the data corresponding to the dominant peaks with approximately the same frequency are recorded and summarized in Tables 68 .

Table 6: Highest Peak Data from S-Transforms of Basalt Fibers Set X

\begin{tabular}{cccc}
\hline Sample & Frequency (MHz) & Time (Millisecond) & Magnitude \\
\hline $10 \mathrm{~J}$ & 0.106 & 0.07132 & 1 \\
20J & 0.108 & 0.06956 & 1.1727 \\
30J & 0.112 & 0.06809 & 0.8111 \\
\hline
\end{tabular}

Again, data from S-Transform plots of the basalt sets $\mathrm{Y}$ and Z, not shown here, are summarized in Tables 7 and 8. In this case, it is observed that the magnitude of the peaks decrease with the increase in impact energy. 
Table 7: Highest Peak Data from S-Transforms of Basalt Fibers Set Y

\begin{tabular}{cccc}
\hline Sample & Frequency (MHz) & Time (Millisecond) & Magnitude \\
\hline 10J & 0.106 & 0.07054 & 1 \\
20J & 0.106 & 0.06494 & 0.62351 \\
30J & 0.110 & 0.06177 & 0.53175 \\
\hline
\end{tabular}

Table 8: Highest Peak Data from S-Transforms of Basalt Fibers Set Z

\begin{tabular}{cccc}
\hline Sample & Frequency (MHz) & Time (Millisecond) & Magnitude \\
\hline 10J & 0.100 & 0.06302 & 1 \\
20J & 0.112 & 0.06748 & 0.86572 \\
30J & 0.112 & 0.06173 & 0.70451 \\
\hline
\end{tabular}

\subsubsection{Time of Flight}

The change in the signal arrival time, or in other words the change in time of flight (TOF), is first investigated. From the S-Transform plots, the variations of the TOF for damaged specimens relative to a reference specimen (which is the least damaged specimen) are plotted in Figures 9 and 10 for the most dominant peaks. The plots show the variations of TOF as the impact energy changes, for all investigated samples.
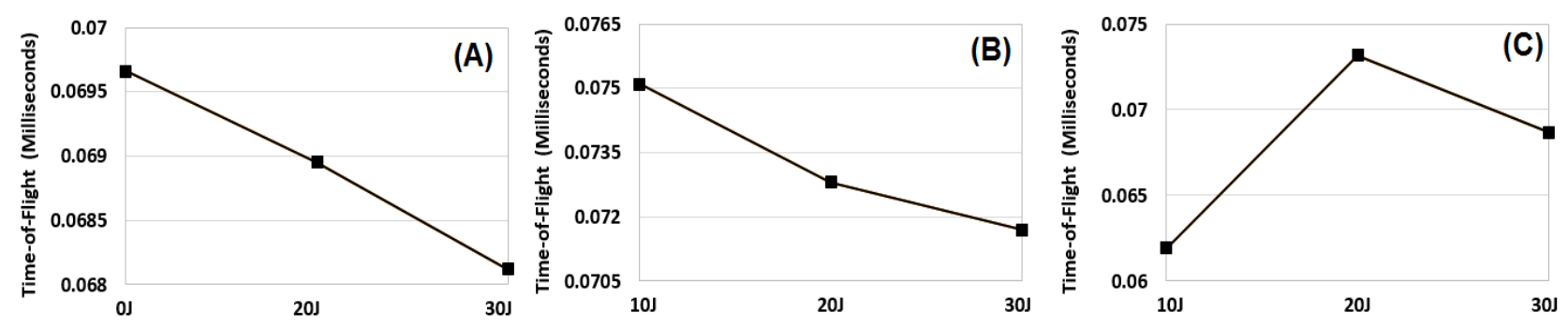

Figure 9: Time-of-Flight Plot of All Glass Fiber Sets

Generally speaking, it can be seen that TOF decreases with the increase of the impact energy. However, sets C and $\mathrm{Z}$ exhibit a trend that is not monotonic, presumably due to the occurring failure mechanisms. 

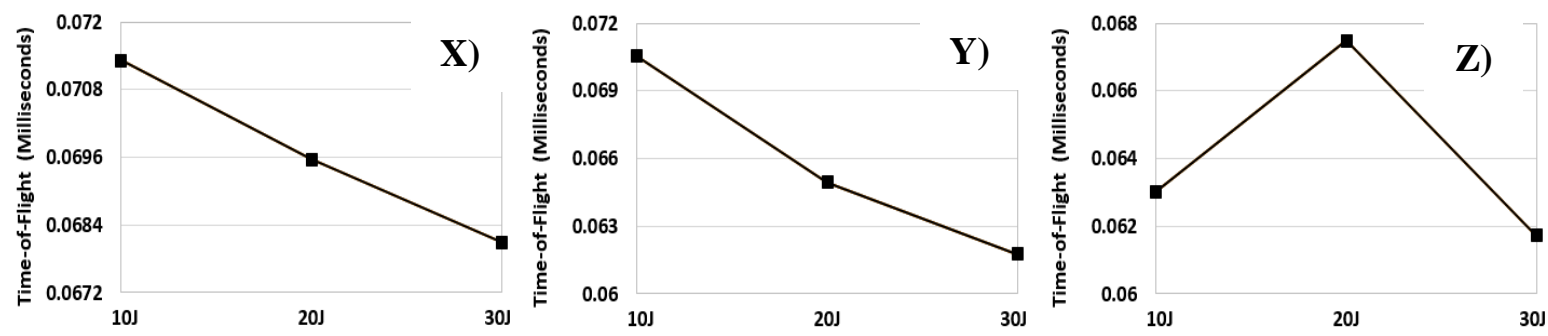

Figure 10: Time-of-Flight Plot of All Basalt Fiber Sets

\subsection{Non-Linear Analysis}

\subsubsection{Sideband Peak Count}

The non-linear analysis of the samples is performed using sideband peak count (SPC). The SPC technique counts the number of peaks in the FFT plots for given threshold values. In general, a linear material shows fewer peaks compared to a non-linear material. Therefore, SPC values for linear and nonlinear materials should be different. The composite plates examined in this study show some non-linear behavior to start with. Increasing impact energy causes more damage in the material, and causes the material more non-linear. Obtaining sideband peak count or SPC values requires relatively simple signal processing tools that are implemented to count the number of peaks observed for a given threshold value. First, the total number of peaks in the FFT plot are counted for all signals shown in Figure. 11. Then, a threshold is set, and the number of peaks below the threshold are counted. Finally, the number of peaks above the threshold are calculated by subtracting the number of peaks below the threshold from the total number of peaks.

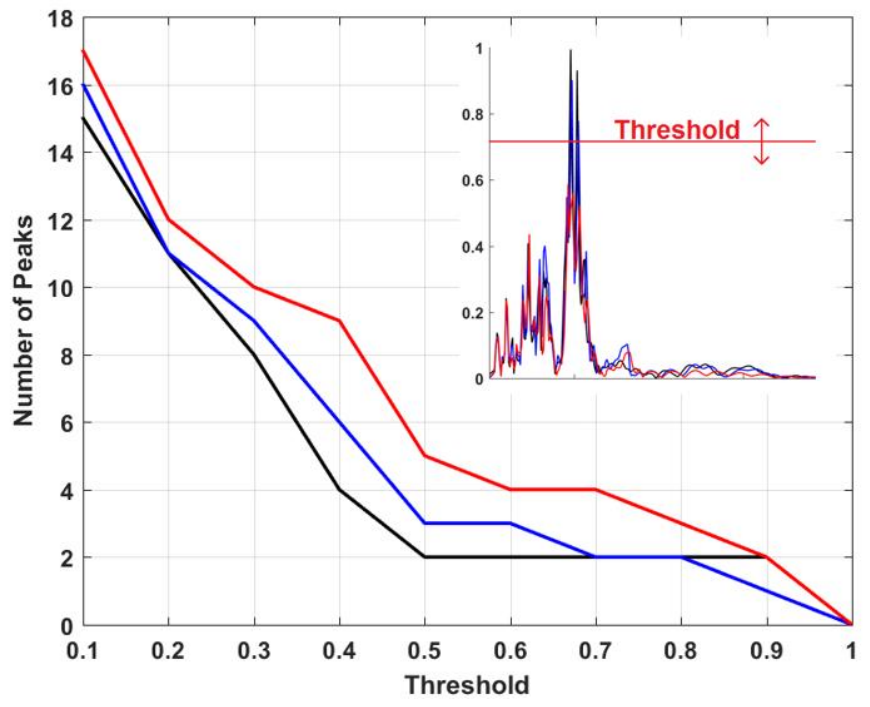

Figure 11: Visualization of FFT and Threshold of SPC

The threshold value can be increased or decreased which is useful for studying the full spectrum of peaks. For example, a threshold value of $20 \%$ will only analyze the peaks that go up to $20 \%$ of the FFT's peak magnitude value. Therefore, peaks at $20 \%$ threshold are 0 since the peaks above $20 \%$ are disregarded. This can be useful in finding the range of peaks that are more influenced by the impact energies. A threshold value of $100 \%$ is always examined.

However, may not always show a clear trend. Figures 12 and 14 show the SPC of both glass and basalt fiber composite sets, respectively, at a threshold value of $100 \%$. In the case of the basalt fibers a trend can be seen for all sets at a threshold of $100 \%$. The least damaged sample generally has a lower peak count than the samples with more damage. 
The sets of glass fibers do not show a clear trend at a threshold of 100\%. Therefore, the SPC plots are again plotted at a threshold of $20 \%$ where a trend is clearer. As explained earlier these changes in threshold values can be due to the different material and different failure mechanism. Further studies are required to select proper thresholds for certain materials or failure mechanisms. The sets B and $\mathrm{C}$ show a trend of increasing peaks for higher impact energies however, set A does not demonstrate the same behavior. At a threshold of 20\% as shown in Figure. 13 the trend can clearly be seen in set A however, it is not as clear in sets B and C.
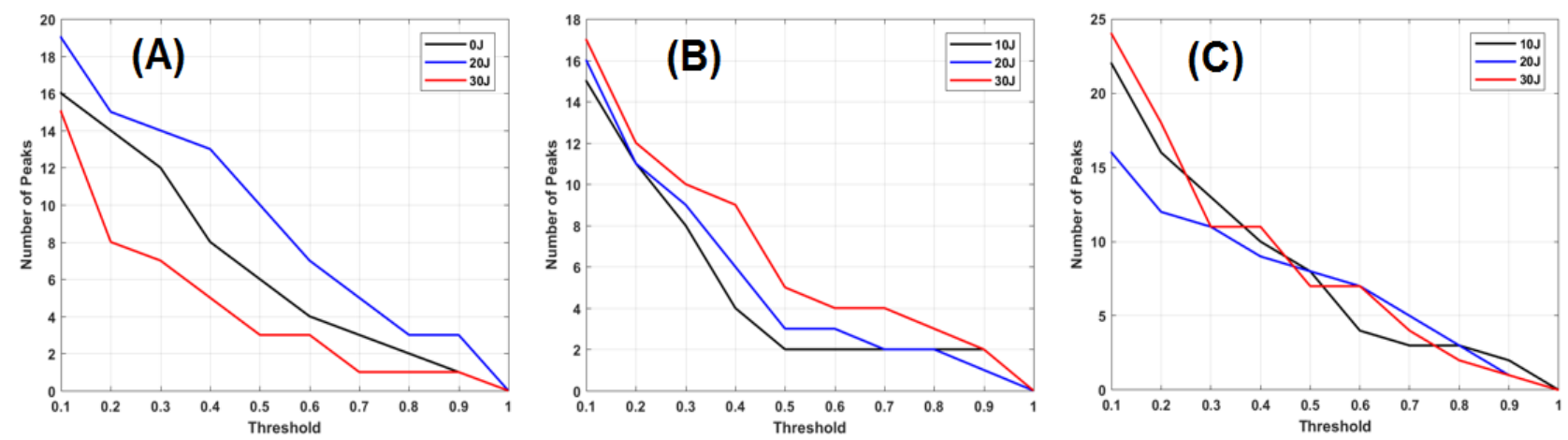

Figure 12: SPC of All Glass Fiber Sets at 100\% Threshold
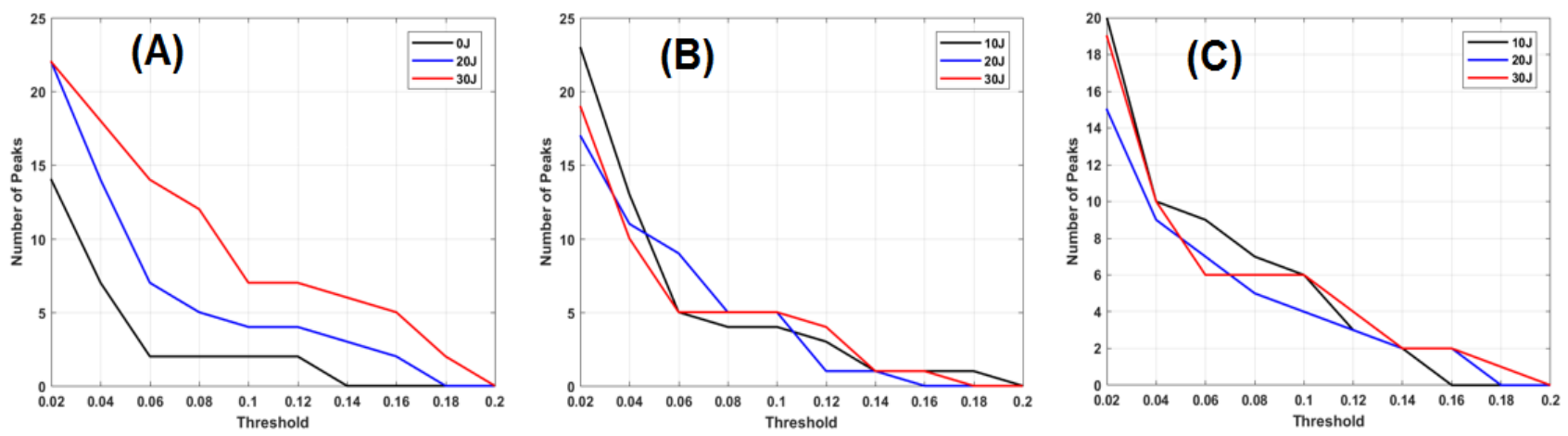

Figure 13: SPC of All Glass Fiber Sets at 20\% Threshold
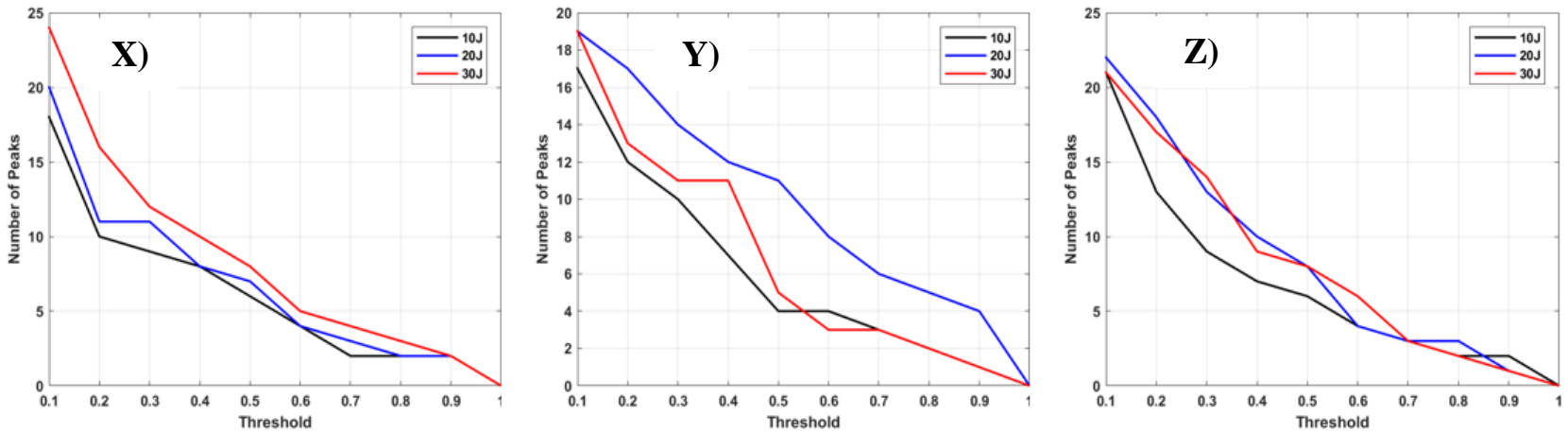

Figure 14: SPC of All Basalt Fiber Sets at 100\% Threshold 


\section{Conclusion}

To conclude it is observed that both linear and non-linear ultrasonic techniques show some promising results in monitoring damages in composite plate specimens. The linear S-Transform based TOF analysis seems to be capable of detecting samples with higher impact energies. The decrease in TOF can be related to the impact energy and induced damage. The TOF technique is advantageous since it is not sensitive to the bonding condition between the transducers and composite plate samples. Furthermore, the non-linear SPC technique shows clear distinctions and trends with varying impact energies. However, more studies are needed to determine which threshold values and what peaks are affected by certain failure mechanisms.

\section{Acknowledgments}

The author acknowledges the financial support of Qatar University (Doha, Qatar)

\section{References}

[1] Kundu, T. (Hg.) (2012): Ultrasonic and electromagnetic NDE for structure and material characterization. Engineering and biomedical applications. Boca Raton, FL: CRC Press.

[2] Kundu, T., Jesús N. Eiras, Weibin Li, Peipei Liu, Hoon Sohn, and Jordi Payá. "Fundamentals of Nonlinear Acoustical Techniques and Sideband Peak Count." In Nonlinear Ultrasonic and Vibro-Acoustical Techniques for Nondestructive Evaluation, pp. 1-88. Springer, Cham, 2019.

[3] Shelke, A., Amjad, U.,Vasiljvic, M., Kundu, T. and Grill, W., "Extracting Quantitative Information on Pipe Wall Damage in absence of clear Signals from Defect," ASME Journal of Pressure Vessel Technology 134, 051502-1 to 051502-7 (2012).

[4] Amjad, U., Chi Hanh Nguyen, S. K. Yadav, E. Mahmoudabadi, and T. Kundu. "Change in time-of-flight of longitudinal (axisymmetric) wave modes due to lamination in steel pipes." In SPIE Smart Structures and Materials+ Nondestructive Evaluation and Health Monitoring, pp. 869515-869515. International Society for Optics and Photonics, (2013)

[5] Amjad, U., Yadav, S.K. and Kundu, T., "Detection and quantification of diameter reduction due to corrosion in reinforcing steel bars," Structural Health Monitoring 14(5), 532-543 (2015).

[6] Amjad, U., Yadav, S. K., and Kundu, T., "Detection and quantification of pipe damage from change in time of flight and phase," Ultrasonics 62, 223-236 (2015).

[7] Amjad, U., Yadav, S.K. and Kundu, T., "Detection and quantification of delamination in laminated plates from the phase of appropriate guided wave modes," Optical Engineering 55(1), 011006 (2015).

[8] Eiras, J.N., Amjad, U., Mahmoudababi, E., Payá, J., Bonilla, M. and Kundu, T. "Monitoring ageing of alkali resistant glass fiber reinforced cement (GRC) using guided ultrasonic waves." Health Monitoring of Structural and Biological Systems 8695, 86950Q (2013).

[9] Aymerich, F, Staszewski, W. J. "Impact damage detection in composite laminates using nonlinear acoustics" Compos Part A: Appl Sci Manuf. 41(9), 1084-1092 (2010).

[10] Liu, Sohn, Kundu, and Yang. "Noncontact Detection of Fatigue Cracks by Laser Nonlinear Wave Modulation Spectroscopy (LNWMS)." NDT and E International 66 (2014): 106-116. 